\title{
Fabrication and Permeability Characteristics of Microdialysis Probe Using Chitosan Nanoporous Membrane
}

\author{
Yun-Ju Chuang, Mei-Jung Chen, and Pei-Ru Chen \\ Department of Biomedical Engineering, Ming Chuan University, 5 De Ming Road, Gui Shan District, \\ Taoyuan County 333, Taiwan \\ Correspondence should be addressed to Pei-Ru Chen; prchen@mail.mcu.edu.tw
}

Received 21 January 2014; Revised 1 May 2014; Accepted 23 May 2014; Published 15 June 2014

Academic Editor: Yitzhak Mastai

Copyright (C) 2014 Yun-Ju Chuang et al. This is an open access article distributed under the Creative Commons Attribution License, which permits unrestricted use, distribution, and reproduction in any medium, provided the original work is properly cited.

In this article, a nanoporous chitosan polymer membrane was successfully produced and applied as microdialysis membrane for in vitro sampling of biomolecules. With the use of nanoparticle leaching technique, porogenic gelatin nanoparticles formed nanopores in the chitosan-based membrane to create a secure implantable nanoporous membrane for biomolecule sampling. The gelatin nanoparticles size was in the range of 45 to $70 \mathrm{~nm}$, and the pore size of the chitosan membrane was around 40 to $100 \mathrm{~nm}$. The porosity of membrane was found to be dependent on the mixing ratio of chitosan solution and gelatin nanoparticles solution. The results of diffusion study showed that we can alter the mixing ratio of porogen to achieve size-selective molecular diffusion, which means that the porosity and cut-off size of porous membrane can be controlled. The recoveries of the probe fabricated from the chitosan-based membrane were examined for four different model compounds of different molecular weights: 2-NBDG, substance P, TNF- $\alpha$, and FITC-BSA. The microdialysis probes showed linear responses and substantial recovery to various concentrations of biomolecules. These results indicated that the microdialysis probe constructed by chitosan nanoporous membrane could sample and monitor the biomolecules in vitro and has the potential for the application in vivo.

\section{Introduction}

Microdialysis perfusion is a minimally invasive method for sampling of biomolecules from organ extracellular fluids in anesthetized or awake animals and human subjects [1]. Such perfusion technique has been a valuable tool for in vivo investigations in the fields of neuroscience, pharmacology, and physiology [2]. The permeable membranes of an implanted microdialysis probe are mainly made of nanoporous materials constructed from polycarbonate, regenerated cellulose, polyethersulfone, or polysulfone. These membranes usually having molecular weight size cut-off ranging from a few $5 \mathrm{kD}$ to $400 \mathrm{kD}$ should ensure appropriate transport of biomolecules while excluding interferents [3]. In addition, the membranes are usually biocompatible and elastic which minimizes the host immune response and exerts flexible and mechanical property. Most of the materials that are used in concurrent home-made or commercial microdialysis probes are nonbiodegradable or reabsorbable. Microdialysis membrane made of biodegradable or reabsorbable materials may have the advantages of long-term in vivo sampling and no additional surgical removal is needed.

Among all of the materials, chitosan was one of the biopolymer conformed foregoing essentials. Chitosan, (1-4)2 -amino-2-deoxy- $\beta$-D-glucan, is the $\mathrm{N}$-deacetylated derivative of chitin and is low cytotoxic, hydrophilic, elastic, biocompatible, and biodegradable materials [4-9]. These unique properties make it an attractive material for biomedical applications.

Porosity of the microdialysis membrane plays a critical role for the efficient diffusion of biomolecules crossing the membrane; several fabrication techniques of porous materials have been developed such as salt leaching, rapid prototyping, electrospinning, and two-phase liquid system [10]. Solvent casting and particulate leaching were widely used in the manufacturing of porous polymer scaffolds. Salt and gelatin submicropowder are the most commonly used particulate for this method because they are known biocompatible and noncytotoxic materials [11]. All of the above techniques produced porous materials only in micrometer 


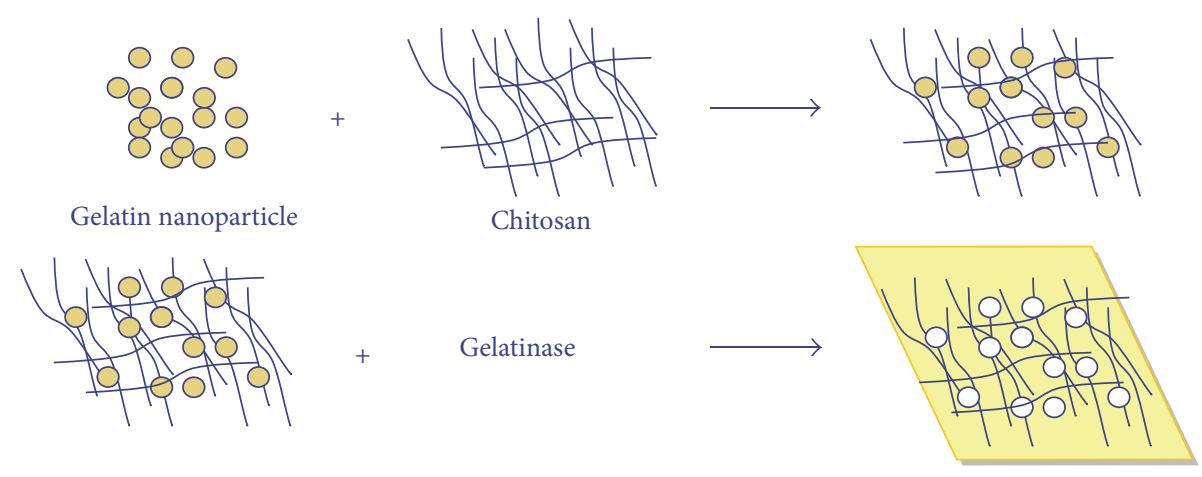

FIGURE 1: Illustration of enzyme digested nanoporous chitosan membrane for long-term implantable biosensing. The gelatin nanoparticle was added in chitosan solution to prepare a thin film and then digested by gelatinase to form a nanoporous membrane.

scale, and the pore size in microdialysis application was too large to exclude interferents.

In this study, we employed nanoparticle leaching technique. The gelatin nanoparticles were designed as porogen to form nanopores in chitosan film. Figure 1 shows a schematic diagram of the formation of nanoporous chitosan membrane. Gelatin nanoparticles were doped into chitosan film, were subsequently etched away by collagenase, and thus leave nanometer sized pores for increasing porosity of the chitosan membrane. The gelatin particle size distribution was determined by particle size analyzer and TEM. The pore size and porosity of membrane were examined by SEM and porosity test, respectively. The permeability and cut-off size characteristics were determined by diffusion study with different molecular weight size of dextran. Also, the nanoporous chitosan membrane was successfully applied to fabricate a microdialysis probe, and in vitro sampling of biomolecules with different molecular weights, 2-NBDG (0.3 kDa), FITC-BSA (65 kDa), TNF- $\alpha$ (17.3 kDa), and substance $\mathrm{P}(1.347 \mathrm{kDa})$, was performed for validation of microdialysis probe constructed by chitosan nanoporous membrane.

\section{Materials and Methods}

2.1. Preparation of Gelatin Nanoparticles. Gelatin nanoparticles were prepared by two-step desolvation method [12]. The size of gelatin nanoparticles can be affected by using different molecular weight of gelatin. In the studies of Saxena et al. [13], the size of the nanoparticles produced by 75 bloom $(22 \mathrm{kDa})$ gelatin is smaller than 300 bloom $(87.5 \mathrm{kDa})$. Therefore, gelatin sample of 75 bloom was chosen for preparing nanoparticles.

Gelatin of $1.25 \mathrm{~g}$ (75 bloom, type B, from bovine skin, Sigma Co., USA) was dissolved in $25 \mathrm{~mL}$ water at $35-37^{\circ} \mathrm{C}$ temperature. In order to achieve the desolvation and rapid sedimentation of the gelatin, $25 \mathrm{~mL}$ of acetone was added to this solution. The supernatant consisted of some desolvated gelatin as well as gelatin in solution and was discarded. The remaining sediment was redissolved in $25 \mathrm{~mL}$ of water under $35-37^{\circ} \mathrm{C}$ temperature and the $\mathrm{pH}$ value of solution was adjusted to 2.5 .
The gelatin was then desolvated again by addition of $25 \mathrm{~mL}$ acetone dropwise. After $10 \mathrm{~min}$ of stirring, glutaraldehyde $500 \mu \mathrm{L}$ (8\%) was added to cross-link the particles. After stirring for another $8 \mathrm{hrs}$, the dispersion was centrifuged at $10,000 \mathrm{~g}$ for $30 \mathrm{~min}$. The particles were purified by threefold centrifugation and redispersion in $35 \mathrm{~mL}$ of acetone/water (30/70). After the last redispersion, the acetone was evaporated on a water bath at $50^{\circ} \mathrm{C}$.

2.2. Preparation of Nanoporous Chitosan Membrane. Chitosan used for preparing nanoporous membrane was purchased from C\&B Co., Taiwan. The molecular weight of chitosan is $300 \mathrm{kDa}$, purity is $95 \%$, degree of deacetylation is more than $95 \%$, and ash content is lower than $0.5 \%$. 2 wt $\%$ chitosan solution (M.W. 300,000 Da) was prepared in $0.1 \mathrm{M}$ acetic acid $(\mathrm{pH}=2.8)$. Adding various volumes of gelatin nanoparticle to chitosan solution formed chitosan/gelatin mixed solution with four mixing ratios (CG 0, CG 25, CG 35 , and CG 50). The CG 0 was nonporous membrane and used as the control group and CG 25, for example, was the membrane prepared with $75 \mathrm{wt} \%$ chitosan and $25 \mathrm{wt} \%$ gelatin nanoparticles. The chitosan/gelatin mixed solution was cast in Petri dish and then dried by heat in oven. Heatdried membranes were prepared by allowing the solvent (water) to evaporate at $50^{\circ} \mathrm{C}$ for $24 \mathrm{hrs}$. Then, all of the dried samples were immersed into $1 \mathrm{M}$ of $\mathrm{NaOH}$ solution for $2 \mathrm{hrs}$. These membranes were removed from $\mathrm{NaOH}$ solution and completely washed by distilled water. Then these membranes were transferred into $0.2 \%$ gelatinase solution at $37^{\circ} \mathrm{C}$ for 24 hrs. Finally, the nanoporous chitosan membranes were lyophilized overnight.

2.3. Equilibrium Water Content and Porosity. Porosity of the porous membranes was estimated from the equilibrium water content. Porosity, $\varepsilon$, is defined by the following equation [14]:

$$
\varepsilon=\frac{\left(V_{t}-V_{\mathrm{mem}}\right)}{V_{t}},
$$

where $V_{t}$ is the total volume of membrane and $V_{\text {mem }}$ is the volume of nonporous phase. 
The equilibrium water content, $Q$, is defined as

$$
Q=\frac{\left(W_{w}-W_{d}\right)}{W_{w}},
$$

where $W_{w}$ is the weight of swollen porous membrane and $W_{d}$ is the weight of dry porous membrane.

If one assumes that most of water is present in pores when the membrane is in equilibrium swelling, the apparent porosity of porous membrane, $\varepsilon$, is represented as in (1). However, the water molecules present in the nonporous phases must be considered. Thus, the porosity of the membrane, $\varepsilon^{\prime}$, was estimated as [14]

$$
\varepsilon^{\prime}=\frac{\left(W_{w}-W_{d}-W_{m \cdot \mathrm{H}_{2} \mathrm{O}}\right)}{W_{w}},
$$

where $W_{m \cdot \mathrm{H}_{2} \mathrm{O}}$ is the amount of water in the nonporous phase. The $W_{m \cdot \mathrm{H}_{2} \mathrm{O}}$ value of each sample is estimated as

$$
W_{m \cdot \mathrm{H}_{2} \mathrm{O}}=\frac{\left(W_{w \cdot \text { non }}-W_{d \cdot \text { non }}\right) W_{d}}{W_{d \cdot \text { non }}},
$$

where $W_{w \cdot \text { non }}$ is the weight of swollen nonporous sample and $W_{d \cdot \text { non }}$ is the weight of dry nonporous sample.

In order to determine the equilibrium water content and porosity of porous membranes, each sample was lyophilized and weighted. The dry samples were weighted first and then immersed into a phosphate buffer $(\mathrm{pH} 7.4)$ until there was no further weight increase. After the excess water on membrane surface was removed by carefully blotting with filter paper, the weights of swollen samples were measured.

2.4. Permeation Experiments. Permeation studies were performed using side-by-side diffusion cells [15] and the experimental setup is shown in Figure 2. Preswollen chitosan membranes were mounted between the two half-cells of the feed cell and receiver cell. A solution with a specific model drug concentration was added to the feed cell, and fresh buffer solution was added to the receiver cell. Fluorescence spectrophotometer was used to measure the fluorescence intensity of the samples taken from the receiver half-cell. The solute concentration of each sample could be determined using a calibration curve derived from the fluorescence of the known concentration of the solute. $3 \mathrm{kDa}, 10 \mathrm{kDa}$, and $40 \mathrm{kDa}$ dextran fluorescein were used as model biomolecules for permeation studies. The receiver compartment was filled with $13 \mathrm{~mL}$ of $\mathrm{pH} 7.4 \mathrm{PBS}$ buffer, while the feed compartment was filled with an equal volume of $0.1 \mathrm{mg} / \mathrm{mL}$ dextran fluorescein solution. At each time point, a $100 \mu \mathrm{L}$ sample solution was taken from the receiver cell, analyzed, and then returned to its compartment. Dextran fluorescein solution was determined by using fluorescence spectrophotometer, the excitation wavelength was $494 \mathrm{~nm}$, and the emission wavelength was $520 \mathrm{~nm}$.

2.5. Fabrication of Microdialysis Probe. The prepared nanoporous chitosan membrane was applied as dialysis membrane for the construction of microdialysis probe. First, the flat

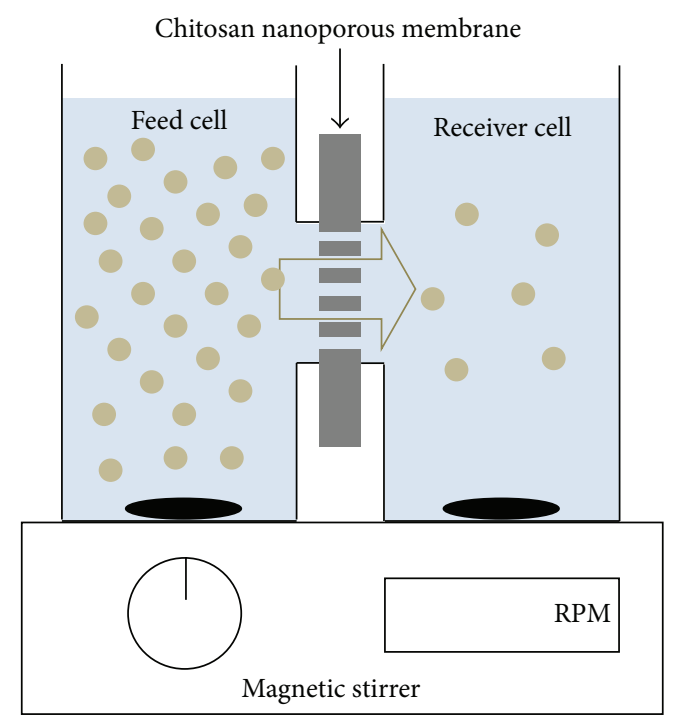

FIGURE 2: Schematic experimental setup for permeation study of nanoporous chitosan membrane. The chitosan membrane was clamped between two half-cells, feed cell and receiver cell. The model biomolecules with specific molecular weight and concentration were added to feed cell and the receiver cell was filled with buffer solution. After beginning of diffusion, the concentration of model biomolecules solution taken from receiver cell was measured.

chitosan membrane was rolled up with a silica fiber to form a hollow shape. The silica fiber acts as a template and its outer diameter and length is $600 \mu \mathrm{m}$ and $8 \mathrm{~mm}$. The rolled-up chitosan membrane was glued on the side and sealed at the tip with polyimide sealing resin (Alltech Associates, Inc., USA). After the sealing resin was fully cured, the silica fiber was drawn out and removed from chitosan membrane, and the hollow shape microdialysis probe made of nanoporous chitosan membrane was formed. Next, inlet and outlet cannula (fused silica capillary) of the desired inner and outer diameter (inner and outer radius are 75 and $180 \mu \mathrm{m}$, resp.) were inserted into the microdialysis probe, advanced to the glued tip. Two $10 \mathrm{~cm}$ pieces of Teflon FEP outflow tubing (CMA/Microdialysis, Solna, Sweden) were attached to the inlet and outlet ports of microdialysis probe to facilitate infused perfusing fluid and dialysate collection, respectively.

2.6. In Vitro Microdialysis Experiments. The microdialysis study was divided into two parts, fluorescence molecules and nonfluorescence molecules.

In the in vitro microdialysis study of fluorescence molecules, the microdialysis system was perfused with $50 \mathrm{mM}$ phosphate buffer solution $(\mathrm{pH} 7.4)$ by a CMA/100 syringe pump with a flow rate of $1 \mu \mathrm{L} / \mathrm{min}$ while immersing the microdialysis probe in analyte solution. The fluorescence microdialysates were allowed to flow through a polyimidecoated fused silica capillary (inner and outer diameter are $250 \mathrm{~m}$ and $360 \mathrm{~m}$ ). A $2 \mathrm{~cm}$ segment of the capillary was stripped off the polyimide coating to serve as the detection window and placed inside a laser-induced fluorescence detector (LIF, Picometrics S. A., Ramonville, France) to analyze the concentration of fluorescence analyte. The experimental 
setup of microdialysis system for fluorescence molecules was shown in Figure 3(a). Two different molecular weight fluorescence molecules, fluorescent glucose analogue (2-NBDG, $0.3 \mathrm{kD}$ ) and fluorescent bovine serum albumin (FITC-BSA, $65 \mathrm{kD}$ ), with three different concentrations were used as analyte to evaluate the recovery. When the microdialysis probe was placed in the analyte solution and perfused at a flow rate of $1 \mu \mathrm{L} / \mathrm{min}$, the analyte concentration in the dialysates gradually increased as a result of analyte crossing the membrane of the microdialysis probe. The analyte concentration in the dialysate was detected and recorded. The experimental procedure was $0-40 \mathrm{~min}$, and microdialysis probes were placed in PBS buffer solution, at $40-80 \mathrm{~min}$ in $31.25 \mu \mathrm{M} 2-\mathrm{NBDG} / 9.6 \mu \mathrm{M}$ FITC-BSA solution, at $80-120 \mathrm{~min}$ in PBS, at $120-160 \mathrm{~min}$ in $62.5 \mu \mathrm{M} 2-\mathrm{NBDG} / 19.2 \mu \mathrm{M}$ FITCBSA solution, at 160-200 min in PBS again, at 200-240 min in $125 \mu \mathrm{M} 2-\mathrm{NBDG} / 38.4 \mu \mathrm{M}$ FITC-BSA, and at $240-280 \mathrm{~min}$ in the PBS buffer solution. The recovery of an analyte is defined as the concentration of this analyte in the dialysate divided by the concentration of this analyte outside the microdialysis probe.

In the in vitro microdialysis study of nonfluorescence molecules, two different molecular weight nonfluorescence molecules, substance P $(1.3 \mathrm{kDa})$ and TNF- $\alpha(17.4 \mathrm{kDa})$, were used as analytes. The experimental setup was shown in Figure 3(b). The microdialysis probes were soaked for at least $40 \mathrm{~min}$ in PBS prior to experimentation. Then the probes were submerged in $2 \mathrm{~mL}$ vial filled with $200 \mathrm{pg} / \mathrm{mL}$ TNF- $\alpha$ or $5 \mathrm{ng} / \mathrm{mL}$ substance $\mathrm{P}$ solution in PBS. The protein and peptide microdialysis sampling were performed at a perfusate flow rate of $1 \mu \mathrm{L} / \mathrm{min}$ with PBS ( $\mathrm{pH}$ 7.4) by a CMA/100 syringe pump. The microdialysates were collected every $40 \mathrm{~min}$, till at $200 \mathrm{~min}$ of experiment the probes were put into PBS to remove proteins and clean probes. The concentration of TNF$\alpha$ and substance $\mathrm{P}$ in the external solution and dialysate was determined by enzyme-linked immunosorbent assay (ELISA) against calibrated standard.

\section{Results and Discussion}

3.1. Structure Characterization of Gelatin Particles and Nanoporous Chitosan Membrane. In this work, gelatin nanoparticles were produced by desolvation method and used as the porogen in particle leaching technique to prepare chitosan nanoporous membrane. The particle size was determined by particle size analyzer (90 Plus, Brookhaven Instruments Co., USA). The morphological assessment of the nanoparticles was carried out by TEM. Figure 4(a) presents the results of particle size analyzer, where $d$ is the diameter of nanoparticles and $G(d)$ is weighting factor that represents the relative percentage contribution of the size range. The largest group has the frequency of 100 . The cumulative undersize distribution, $C(d)$, is the percent of the size distribution at or below the diameter $d$. It is calculated by dividing the sum of the $G(d)$ values at and below $d$ by the total sum of $G(d)$. The $y$-axis unit in the plot of $d$ versus $G(d)$ is arbitrary unit and maximum of $y$-axis is 100 . The $y$-axis unit in the plot of $d$ versus $C(d)$ is percentage. In the Figure 4(a), the results from particle size analyzer showed that the sizes of gelatin particles formed by desolvation are in the range of $48-68 \mathrm{~nm}$. In the TEM and SEM micrograph, shown in Figures 4(b) and 4(c), the morphology of gelatin nanoparticle was observed to be spherical and polydispersed, and the particle size is in the range of 30 to $50 \mathrm{~nm}$ which is in coincidence with the results from particles analyzer. Furthermore, the size distribution of synthesized particles was narrow and the major size is between 48 and $54 \mathrm{~nm}$. The narrow size distribution can be attributed to the use of twice desolvation that can greatly reduce the production of nanoparticles with larger size.

Three kinds of mixing ratio of chitosan and porogen (gelatin nanoparticles) were used to prepare porous chitosan membrane with different porosity, CG 25, CG 35, and CG 50. The scanning electron microscope images of surface and cross section view of nanoporous membrane with three different weight mixing ratios of chitosan and gelatin nanoparticles were shown in Figure 5. The analyzed results showed that the pore size of the nanoporous chitosan membrane was around 40 to $100 \mathrm{~nm}$ which is coincident with the size of gelatin nanoparticles. The pore size range was much smaller than produced by other fabrication techniques of porous materials. For example, Thomson et al. [16] used solvent casting and particulate leaching to render porous materials with $23-52 \mu \mathrm{m}$ of pore diameter. In the Whang et al. [17] studies, the pore size was in the range of $16-32 \mu \mathrm{m}$ produced by emulsion freeze-drying method. The largest pores, $125-150 \mu \mathrm{m}$, were fabricated by rapid prototyping technique in Roy et al. [18] studies. The purpose of porosity in microdialysis probe was to select cytokine transportation; therefore, the microscale pores were too large to exclude interferents. However, the number density of nanopores showed that there is no significant difference between three kinds of nanoporous chitosan membrane. This is due to the dehydration of membrane during drying process and then the pores of membrane were synaeresis. Therefore, the demonstration of porosity and characteristics of the nanoporous chitosan membrane should combine the results of water content and the permeability evidence.

\subsection{Equilibrium Water Content and Porosity of Nanoporous} Chitosan Membrane. When the membranes were totally immersed in water, the spaces of pores were occupied by water. Therefore, the water content was the simple technique to evaluate the porosity of membrane. As shown in Table 1, the water content was increased with the increase of proportion of porogen. When the amount of gelatin nanoparticles was arisen to $50 \%$, the equilibrium water content was arisen to $82.37 \%$ on average.

Based on (3) and (4), the porosity of nanoporous membrane was calculated and shown in Figure 6. The results showed that the porosity was greatly affected by the amounts of the gelatin nanoparticle. The porosity was arisen to $38 \%$ when the mixing ratio of porogen was increased to $50 \%$, but in CG 35 and CG 25 there was no significant difference in porosity evaluation. It may be because some gelatin nanoparticles which situated at the inside of the membrane failed to be digested when treated with collagenase. 


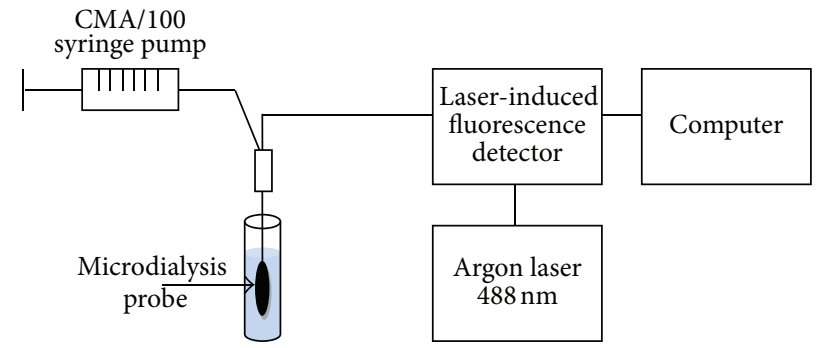

(a)

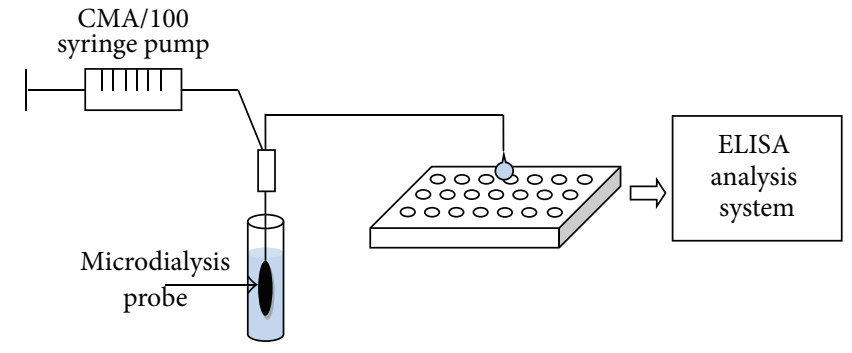

(b)

Figure 3: Schematic experimental setup of microdialysis system for (a) fluorescence molecules and (b) nonfluorescence molecules. In the microdialysis of fluorescence molecules, the dialysates were directly online detected by laser-induced fluorescence detector, while the nonfluorescence dialysates were offline detected by ELISA reader.

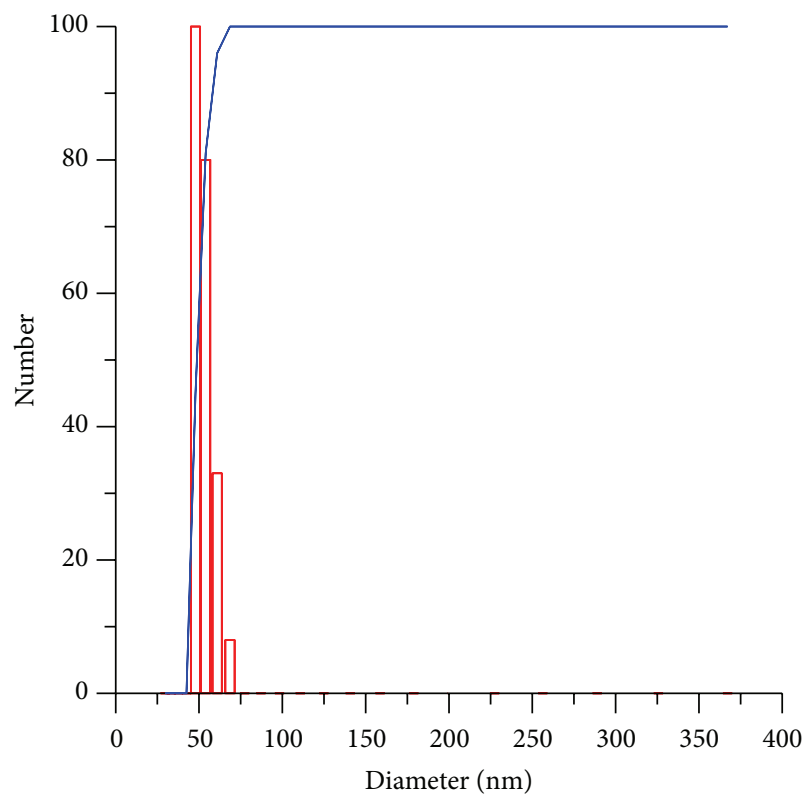

\begin{tabular}{|ccc|ccc|}
\hline$d(\mathrm{~nm})$ & $G(\mathrm{~d})$ & $C(\mathrm{~d})$ & $d(\mathrm{~nm})$ & $G(\mathrm{~d})$ & $C(\mathrm{~d})$ \\
\hline 29.7 & 0 & 0 & 110.8 & 0 & 100 \\
33.4 & 0 & 0 & 124.9 & 0 & 100 \\
37.7 & 0 & 0 & 140.8 & 0 & 100 \\
42.5 & 0 & 0 & 158.7 & 0 & 100 \\
47.9 & 100 & 45 & 178.9 & 0 & 100 \\
54.0 & 80 & 81 & 201.7 & 0 & 100 \\
60.9 & 33 & 96 & 227.4 & 0 & 100 \\
68.6 & 8 & 100 & 256.3 & 0 & 100 \\
77.4 & 0 & 100 & 289.0 & 0 & 100 \\
87.2 & 0 & 100 & 325.7 & 0 & 100 \\
98.3 & 0 & 100 & 367.2 & 0 & 100 \\
\hline
\end{tabular}

(a)

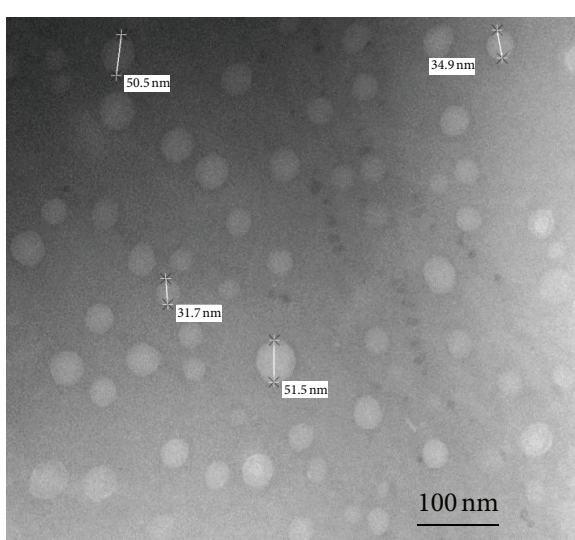

(b)

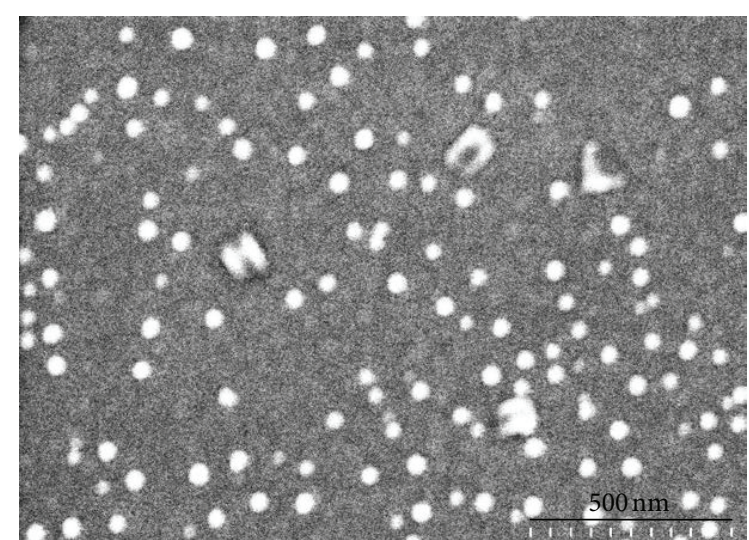

(c)

Figure 4: The size distribution and the morphology of gelatin nanoparticles were analyzed by (a) particle size analyzer, (b) transmission electron microscopy, and (c) scanning electron microscopy. 


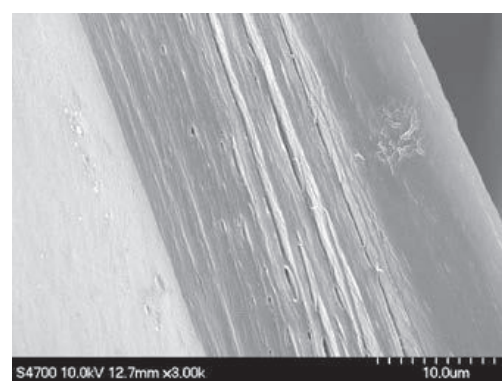

(a)

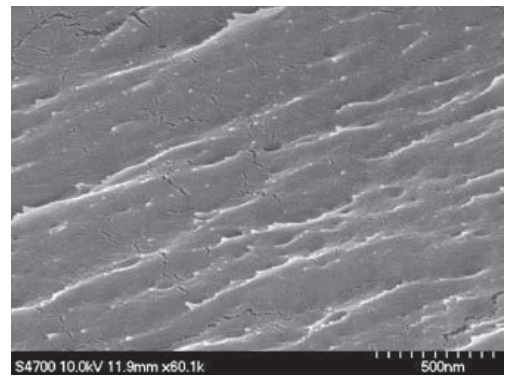

(d)

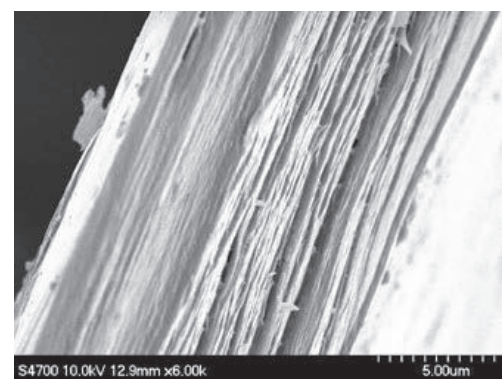

(b)

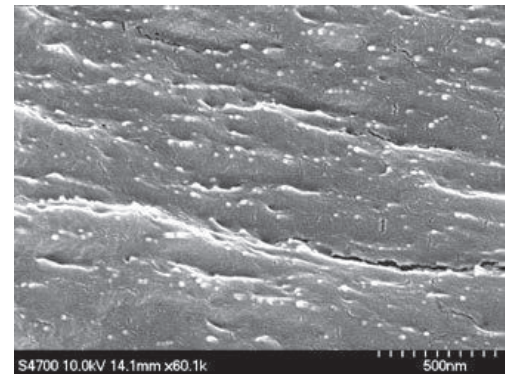

(e)

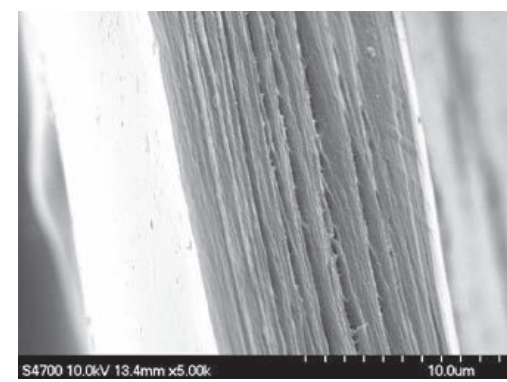

(c)

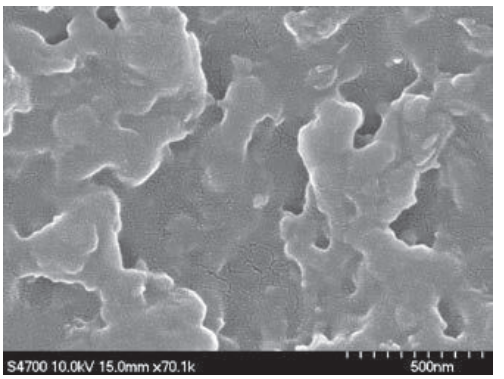

(f)

FIGURE 5: The morphology of cross section (a), (b), and (c) and surface (d), (e), and (f) of the enzyme digested nanoporous chitosan membrane observed by SEM. (a) and (d) were CG 25; (b) and (e) were CG 35; (c) and (f) were CG 50.

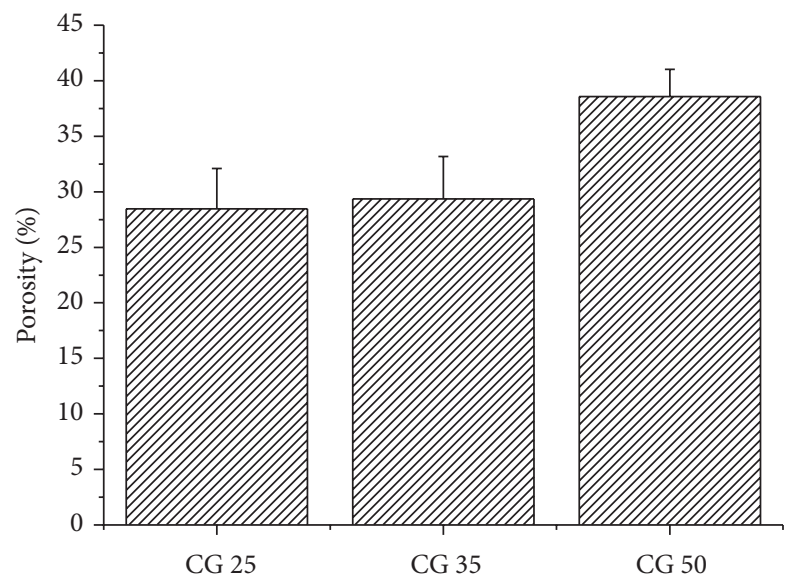

FIGURE 6: The porosity of enzyme digested nanoporous chitosan membrane.

Several studies have emphasized the need for high porosity and high surface area-to-mass ratio for ensuring uniform cell delivery and tissue ingrowth $[19,20]$. Need has also been expressed for polymeric scaffolds to possess an open pore network for optimal diffusion of nutrients and waste [21, 22]. However, Goldstein et al. [23] have cautioned that polylactic-polyglycolic acid (PLG) scaffolds be prepared with a porosity not exceeding $80 \%$ for implantation as it would otherwise compromise the integrity of the scaffold. Thus, in the case of polymeric scaffolds, there may be a conflict between optimizing the porosity and maximizing mechanical properties. Thus, in view of these contradictory factors, there is a need to optimize materials based on their
TABLE 1: The water content of enzyme digested nanoporous chitosan membrane with different porosity controlled by the mixing ratio of chitosan and gelatin nanoparticle.

\begin{tabular}{|c|c|c|c|}
\hline \multicolumn{4}{|c|}{$1 \times 1 \mathrm{~cm}^{2}$ at $25^{\circ} \mathrm{C}, \mathrm{pH} 7.4$ (the data were expressed as mean \pm S.D.) } \\
\hline & Dry weight (mg) & Wet weight (mg) & Water content (\%) \\
\hline 10 & 2.476 & 8 & \\
\hline Chit & $2.294 \pm 0.163$ & 10. & 77. \\
\hline Chit65/gel35 & $2.554 \pm 0.064$ & $10.598 \pm 0$ & $75.90 \pm 11.26$ \\
\hline Chit50/gel50 & $2.270 \pm 0.089$ & $11.604 \pm 0.342$ & $80.43 \pm 3.86$ \\
\hline
\end{tabular}

specific mechanical requirements balanced with their desired useful life and diffusion characteristics. One possible way of achieving this would be to optimize porosity with respect to biomolecules diffusion and match it with biomaterials that can provide adequate mechanical properties. Particulate leaching is a popular technique used for fabricating scaffolds, wherein the pore size of the resulting scaffold is controlled by the size of the porogen, and porosity is controlled by the porogen/polymer ratio. However, natural porogen dispersion allows little control over pore interconnectivity and this has led to the modification of this technique to produce greater pore interconnectivity.

3.3. Diffusion Characteristics and Cut-Off Size of Nanoporous Chitosan Membrane. To determine the permeability of $3 \mathrm{kDa}, 10 \mathrm{kDa}$, and $40 \mathrm{kDa}$ dextran, the nanoporous chitosan membrane was clamped in a diffusion cell, with a cross sectional area for transport. In Figure 7(a), after diffusion for $52 \mathrm{hrs}$, the results of permeability of $3 \mathrm{kDa}, 10 \mathrm{kDa}$, and $40 \mathrm{kDa}$ dextran of nonporous chitosan membrane were 


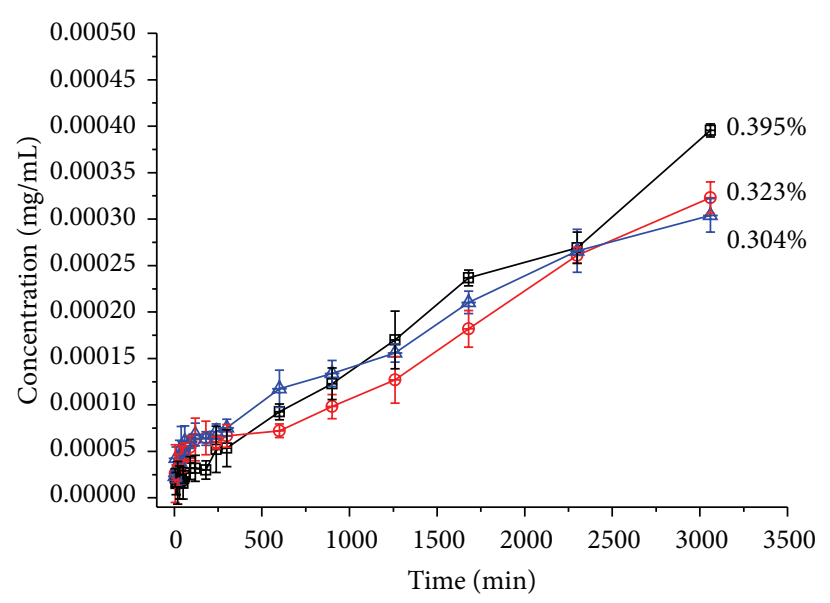

(a)

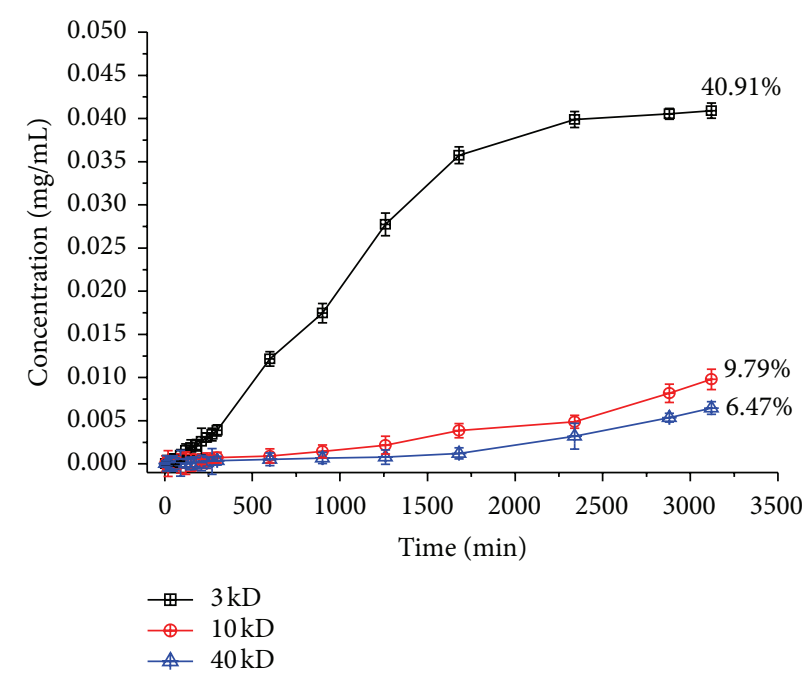

(c)

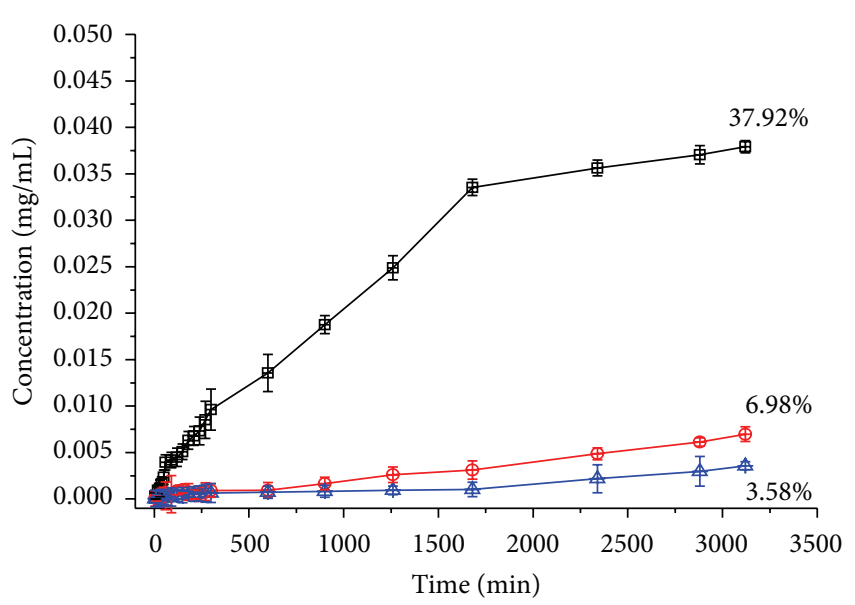

(b)

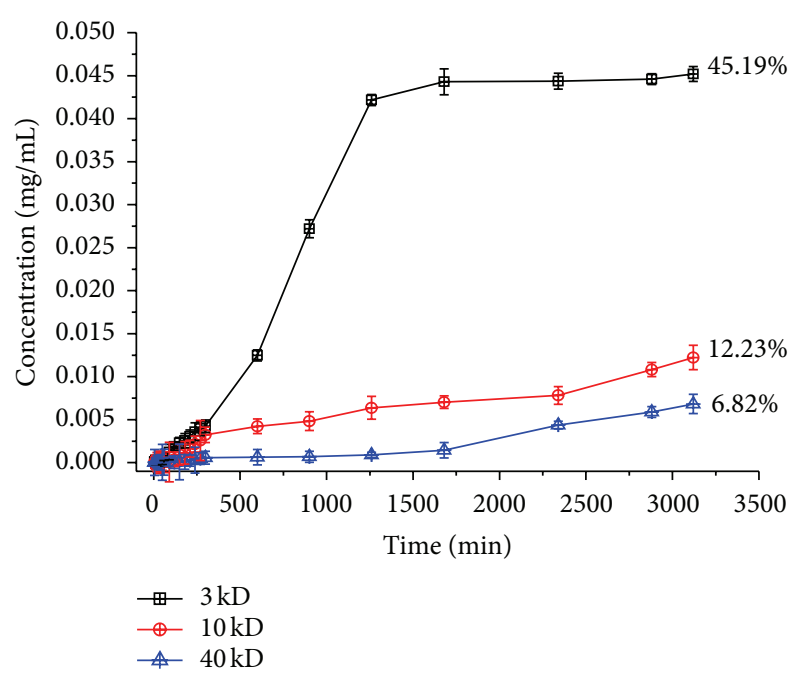

(d)

Figure 7: Permeability of $3 \mathrm{kDa}, 10 \mathrm{kDa}$, and $40 \mathrm{kDa}$ dextran fluorescein molecules across porous chitosan membrane with different weight mixing ratio of chitosan and gelatin nanoparticles. (a) CG 0; (b) CG 25; (c) CG 35; (d) CG 50.

only $0.395 \%, 0.323 \%$, and $0.303 \%$ transported to acceptor compartment. Comparatively, the nanoporous chitosan membranes, CG 25, CG 35, and CG 50 , digested by $0.2 \%$ collagenase overnight showed a fast diffusion of $3 \mathrm{kDa}$ dextran (Figures 7(b), 7(c), and 7(d)). There were $37.92 \%$ of total dextran that diffused or traversed the CG 25 membrane after $28 \mathrm{hrs}$ of permeation study. When the ratio of porogen arose to $50 \%$, the permeability of $3 \mathrm{kDa}$ dextran could be up to $44.29 \%$ after $28 \mathrm{hrs}$.

In addition, we used the permeability in $600 \mathrm{~min}$ to evaluate the cut-off size of nanoporous membrane shown in Table 2. After 600 min of permeation study, the diffused amount of $3 \mathrm{kDa}$ dextran was reached not only to $12.2-13.5 \%$ through nanoporous membrane, but also to $0.09 \%$ through nonporous membrane. In cut-off size evaluation, $10 \mathrm{kDa}$ dextran could traverse the nanoporous chitosan membrane when the porogen adding amount increased to $50 \%$, but there was significantly less dextran diffused through CG 35, CG 25, and CG 0 membranes.
TABLE 2: Cut-off size in diffusion study (data were expressed as mean \pm S.D.).

\begin{tabular}{lccc}
\hline & 3 kD dextran & 10 kD dextran & $40 \mathrm{kD}$ dextran \\
\hline CG 50 & $12.48 \pm 0.9 \%$ & $4.53 \pm 0.85 \%$ & $0.65 \pm 0.64 \%$ \\
CG 35 & $12.17 \pm 0.84 \%$ & $0.92 \pm 0.74 \%$ & $0.54 \pm 0.44 \%$ \\
CG 25 & $13.55 \pm 2.2 \%$ & $0.95 \pm 0.83 \%$ & $0.73 \pm 0.65 \%$ \\
CG 0 & $0.09 \pm 0.01 \%$ & $0.08 \pm 0.01 \%$ & $0.13 \pm 0.02 \%$ \\
\hline
\end{tabular}

Permeation is the movement of molecules by diffusion from high concentration to low concentration through a semipermeable membrane. Only those molecules that are small enough to fit through the membrane pores are able to move through the membrane and reach equilibrium with the entire volume of solution like $3 \mathrm{kDa}$ dextran in this system (Figure $7(\mathrm{~d})$ ). Once equilibrium is reached, there is no further net movement of the substance because molecules will be moving through the pores into and out of 


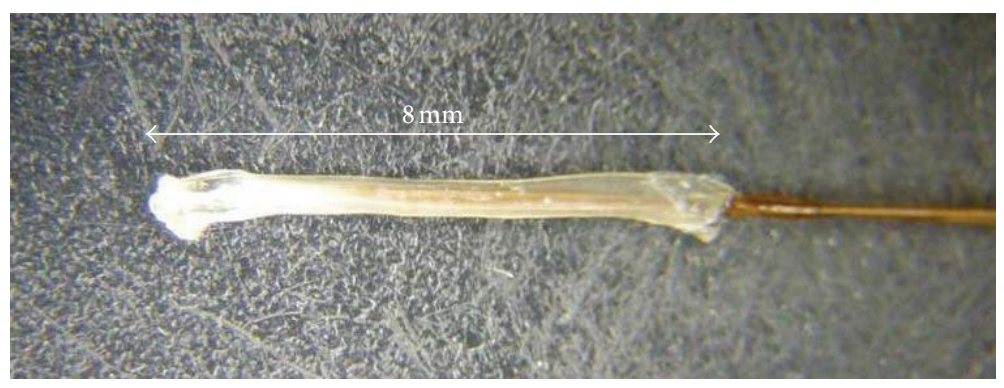

(a)
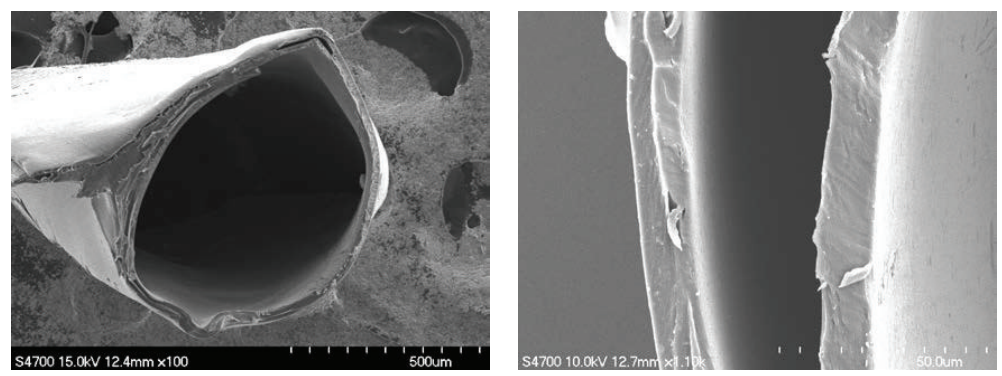

(b)

FIGURE 8: (a) The photo image of microdialysis probe with inlet and outlet fused silica capillary and the length of microdialysis probe is about $8 \mathrm{~mm}$. (b) The cross section image of microdialysis probe observed by SEM. The inner diameter of the probe was about $650 \mu \mathrm{m}$, the outer diameter was about $720 \mu \mathrm{m}$, and the thickness of membrane was about $10 \mu \mathrm{m}$.

the membrane at the same rate. By contrast, large molecules, such as $40 \mathrm{kDa}$ dextran, that are less capable of passing through the membrane pores will remain on the same side of the membrane as they were when diffusion was initiated. In addition, the pore interconnectivity in membrane must be concerned with. An increase in porosity leads to an increase in permeability when the pores are highly interconnected.

However, the dextran has the characteristic of water absorption, and then the cut-off size evaluation in Table 2 was only proper for this kind of molecules diffused study. Furthermore, water is such a small molecule that it is capable of passing through the pores of virtually all dialysis membranes. When dialyzing a high solute concentration or large molecule against a dilute dialysis buffer, there will be a net movement of water (and possibly salts) into the membrane. Glycerol and some carbohydrates such as dextran are especially hygroscopic and also significantly affect the osmosis of water across the membrane [24]. This phenomenon may cause a change in volume of the sample and it is considerable during permeation studies.

3.4. In Vitro Microdialysis Study. The chitosan nanoporous membrane with 50/50 mixing ratio of chitosan/gelatin (CG 50) was successfully fabricated to become a microdialysis probe. The photo image of fabricated microdialysis probe was shown in Figure 8(a) and the length of microdialysis probe is about $8 \mathrm{~mm}$. The SEM image of cross section view of microdialysis probe was shown in Figure 8(b). From the SEM image, we can find that the inner and outer diameter of microdialysis probe are about 650 and $720 \mu \mathrm{m}$ and the thickness of microdialysis membrane is about $10 \mu \mathrm{m}$.
The sampling capability of our microdialysis probe for 2NBDG and FITC-BSA was validated by online laser-induced fluorescence analysis system and the results were shown in Figure 9. Three concentrations of $2-\mathrm{NBDG}$ standard solutions were tested $(31.25,62.5$, and $125 \mu \mathrm{M})$ as shown in Figure 9(a). When the microdialysis probe was placed in the standard solution and perfused at a flow rate of $1 \mu \mathrm{L} / \mathrm{min}$, the $2-\mathrm{NBDG}$ concentration in the perfusates gradually increased as a result of glucose crossing the membrane of the microdialysis probe. Approximately $20 \mathrm{~min}$ after the microdialysis, the 2-NBDG concentration has reached maximum. Then the microdialysis probe was placed into PBS solution to wash the microdialysis and the fluorescence intensities indicated that almost all 2NBDG was washed out from the inside of probe. However, in the FITC-BSA test as shown in Figure 9(b), the concentration could not reach a plateau in the same interval because the high molecular weight needed longer time to reach equilibrium. Relatively, the FITC-BSA was more difficult than $2-\mathrm{NBDG}$ to clean up in microdialysis probe. The recovery of 10 microdialysis probes for 2-NBDG and FITC-BSA is shown in Figures 9(c) and 9(d). The recovery of $2-\mathrm{NBDG}$ and FITC-BSA was $26.5 \%$ and $1.77 \%$ on average, respectively. The large difference in recovery comes from two factors. First, the molecular weight of FITC-BSA $(65 \mathrm{kDa})$ is greatly larger than $2-\mathrm{NBDG}(0.3 \mathrm{kDa})$ and this also means that the size of FITC-BSA is greatly larger than 2-NBDG. Second, the cutoff size of CG 50 membrane is between $10 \mathrm{kDa}$ and $40 \mathrm{kDa}$. Therefore, the pores in microdialysis membrane allow the 2NBDG molecules to pass through easily than FITC-BSA.

In vitro validation of the constructed microdialysis was also performed by selection of nonfluorescent biomolecules 


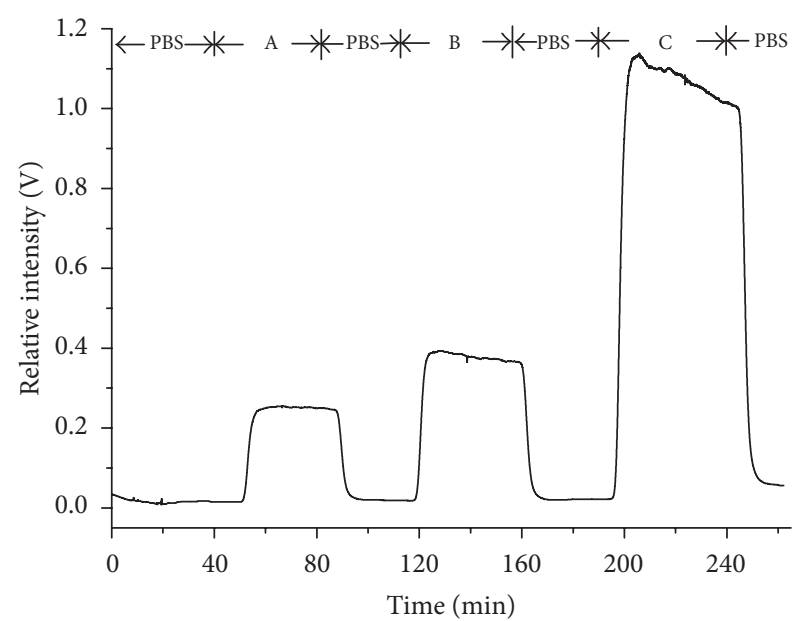
A: $31.25 \mu \mathrm{M}$
B: $62.5 \mu \mathrm{M}$
C: $125 \mu \mathrm{M}$

(a)

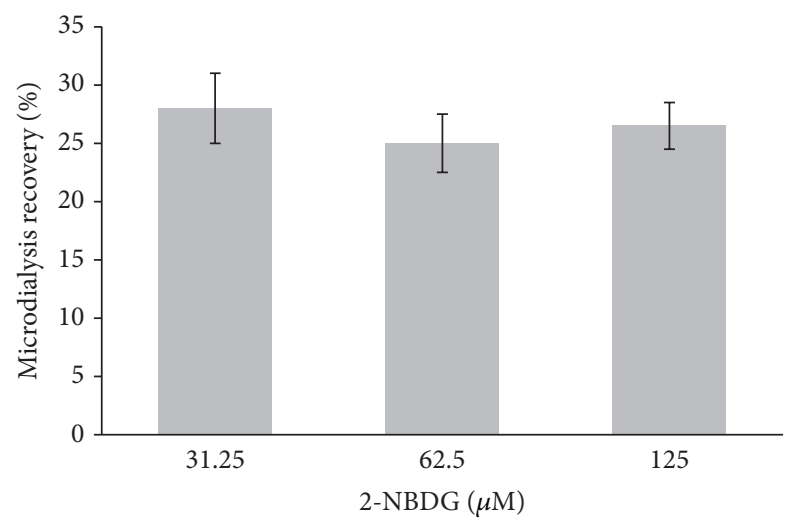

(c)

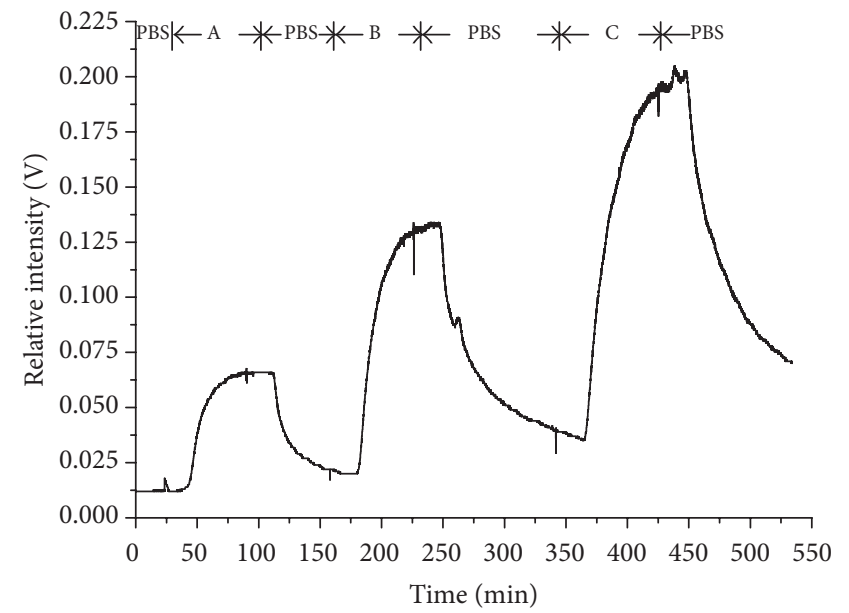
A: $9.6 \mu \mathrm{M}$
B: $19.2 \mu \mathrm{M}$
C: $38.4 \mu \mathrm{M}$

(b)

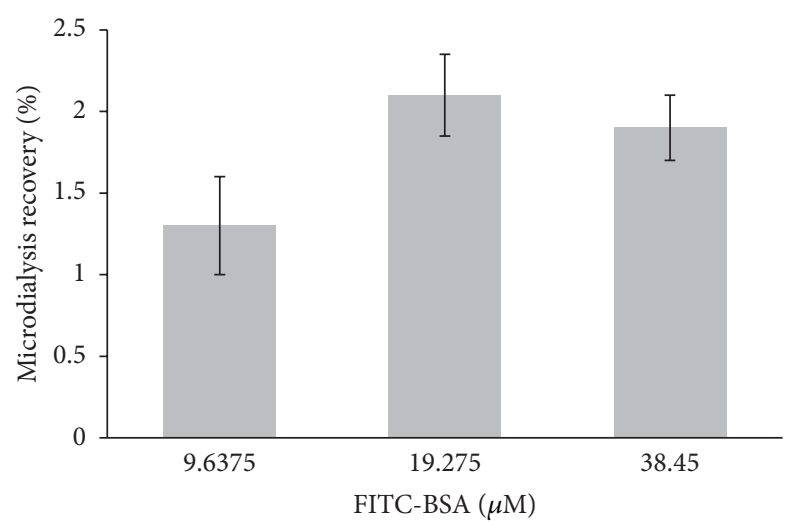

(d)

FIGURE 9: Online flow microdialysis of fluorescence molecules (a) for 2-NBDG and (b) for FITC-BSA. (c) and (d) are recovery of 10 microdialysis probes for 2-NBDG and FITC-BSA, respectively.

at two different sizes, TNF- $\alpha(17.3 \mathrm{kDa})$ and substance $\mathrm{P}$ $(1.347 \mathrm{kDa})$, through the offline analysis with ELISA system. In Figure 10, the sampling capability of our microdialysis membrane for TNF- $\alpha$ and substance $\mathrm{P}$ was validated in vitro at various probes. When the microdialysis probe was placed in $200 \mathrm{pg} / \mathrm{mL}$ TNF- $\alpha$ solution and perfused at a flow rate of $1 \mu \mathrm{L} / \mathrm{min}$, the TNF- $\alpha$ concentration in the perfusates gradually increased as a result of TNF- $\alpha$ crossing the membrane of microdialysis probe. Approximately $120 \mathrm{~min}$ after the perfusion, the TNF- $\alpha$ concentration reached a maximum, which reflected attainment of concentration equilibrium between the inside and outside of the microdialysis probe. The microdialysis probe was then placed in PBS to wash it, and the decreased concentration indicated that almost all TNF- $\alpha$ was displaced from the inside of microdialysis probe. Moreover, different microdialysis probes made in house have the similar performance in TNF- $\alpha$ dialysate. The recovery rates of chitosan-based membrane were considered prominent for both samples, especially for TNF- $\alpha$. The recovery rates of TNF- $\alpha$ and substance $\mathrm{P}$ were $2.87 \%$ and $5.28 \%$ on average. Relatively, TNF- $\alpha$ was more difficult to cross microdialysis membrane than substance $\mathrm{P}$ because of the higher molecular weight. These results indicated that the stability of the microdialysis probe made in house was reliable, whether in TNF- $\alpha$ dialysate or in substance P.

Factors that affect the completeness of dialysis include (1) dialysis buffer volume, (2) buffer composition, (3) time, (4) temperature, and (5) particle size versus pore size. Substances that are very much smaller than the pore size will reach equilibrium faster than substances that are only slightly smaller than the pores. The nanoporous membranes produced by nanoparticle leaching technique contain convoluted pores, not the tube-like pores often found in traditional dialysis tubing. Convoluted pores allow small molecules to pass 


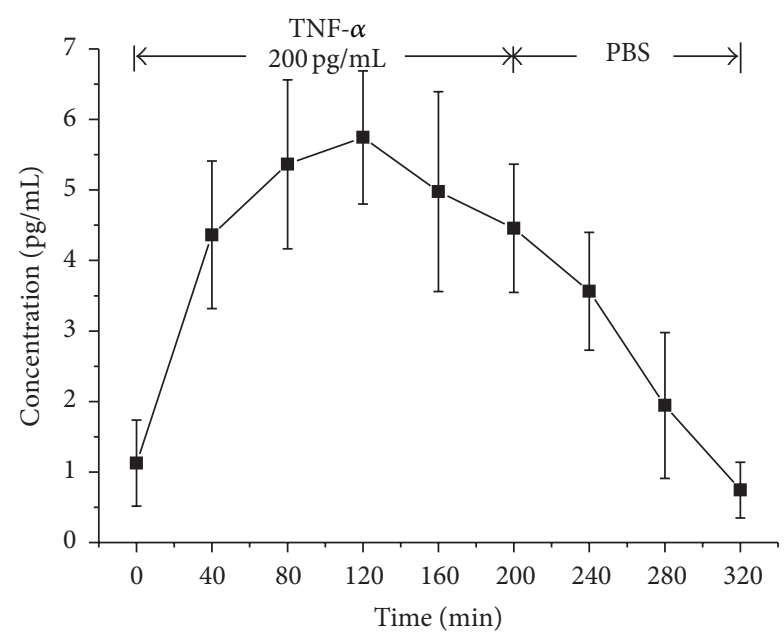

(a)

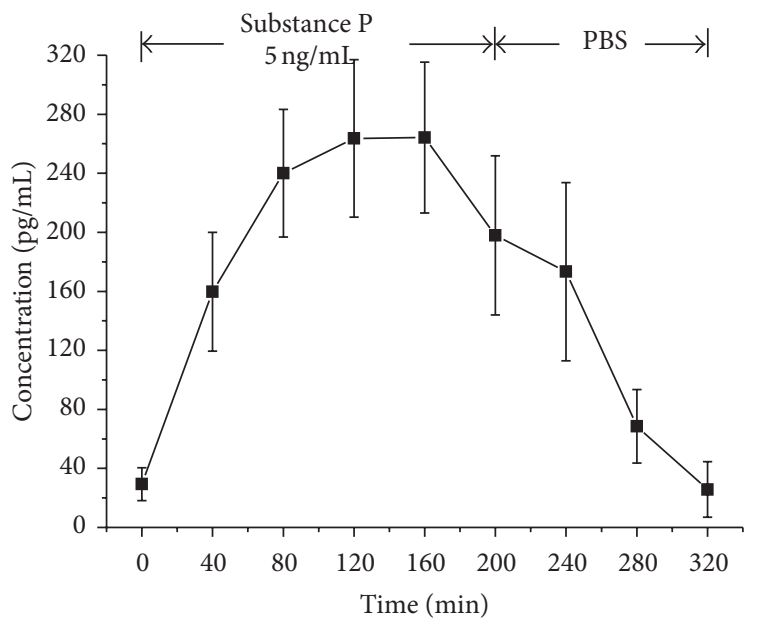

(b)

FIGURE 10: In vitro microdialysis test for TNF- $\alpha$ (a) and substance P (b) with ten probes, respectively. The recovery of TNF- $\alpha$ and substance $P$ was $2.87 \%$ and $5.28 \%$ on average.

through the twists and turns of the pores with greater ease than do larger molecules. However, some molecules form large aggregates and so do not dialyze efficiently despite having low molecular weights. In addition, some molecules may stick to the dialysis membrane. This nonspecific binding can result in sample loss [17]. Finally, the large standard deviation of recovery in different microdialysis probe was because the effective dialysis area of handmade probe was difficult to maintain the same precisely.

\section{Conclusions}

In summary, this study has clearly demonstrated the feasibility of the preparation of nanoporous membrane by gelatin nanoparticles leaching technique. The gelatin nanoparticles were designed as porogen to form nanopores in chitosan polymer membrane. The gelatin nanoparticles were subsequently digested by collagenase and thus leave nanosized pores for increased porosity of the chitosan membrane. The pore size of the chitosan membrane was less than $100 \mathrm{~nm}$, and the porosity could attain to $38 \%$. The results of diffusion study demonstrated that we can alter the mixing ratio of porogen to achieve size-selective substance diffusion that means that the porosity and cut-off size of porous membrane can be controlled.

The microdialysis probes were constructed through the use of nanoporous chitosan polymer membrane. The dialysis results demonstrated the feasibility of chitosan nanoporous membrane for in vitro sampling of biomolecules. Furthermore, the nanoporous membrane is composed of chitosan and gelatin; both are known biocompatible and noncytotoxic material for biomedical applications. Therefore, this nanoporous chitosan membrane has great potential to be applied on implantable microdialysis probe that provided a chamber to monitor biomolecule when injured tissue secreted or to react with allochthonous chemicals in an animal model after ischemia.

\section{Conflict of Interests}

The authors declare that there is no conflict of interests regarding the publication of this paper.

\section{Acknowledgment}

The authors would like to thank the Ministry of Science and Technology, Taiwan, for the financial support under the Project no. NSC 102-2221-E-130-022.

\section{References}

[1] G. Lombardi and F. Moroni, "GM1 ganglioside reduces ischemia-induced excitatory amino acid output: a microdialysis study in the gerbil hippocampus," Neuroscience Letters, vol. 134, no. 2, pp. 171-174, 1992.

[2] N. Wisniewski and N. Torto, "Optimisation of microdialysis sampling recovery by varying inner cannula geometry," Analyst, vol. 127, no. 8, pp. 1129-1134, 2002.

[3] M. Gerritsen, A. Kros, V. Sprakel, J. A. Lutterman, R. J. M. Nolte, and J. A. Jansen, "Biocompatibility evaluation of sol-gel coatings for subcutaneously implantable glucose sensors," Biomaterials, vol. 21, no. 1, pp. 71-78, 2000.

[4] X.-G. Chen, L. Zheng, Z. Wang, C.-Y. Lee, and H.-J. Park, "Molecular affinity and permeability of different molecular weight chitosan membranes," Journal of Agricultural and Food Chemistry, vol. 50, no. 21, pp. 5915-5918, 2002.

[5] Y. I. Cho, H. K. No, and S. P. Meyers, "Physicochemical characteristics and functional properties of various commercial chitin and chitosan products," Journal of Agricultural and Food Chemistry, vol. 46, no. 9, pp. 3839-3843, 1998.

[6] M. Ito, Y. Hidaka, M. Nakajima, H. Yagasaki, and A. H. Kafrawy, "Effect of hydroxyapatite content on physical properties and 
connective tissue reactions to a chitosan-hydroxyapatite composite membrane," Journal of Biomedical Materials Research, vol. 45, pp. 204-208, 1999.

[7] X.-P. Wang, Z.-Q. Shen, F.-Y. Zhang, and Y.-F. Zhang, "A novel composite chitosan membrane for the separation of alcoholwater mixtures," Journal of Membrane Science, vol. 119, no. 2, pp. 191-198, 1996.

[8] F. Shahidi, J. K. V. Arachchi, and Y.-J. Jeon, "Food applications of chitin and chitosans," Trends in Food Science and Technology, vol. 10, no. 2, pp. 37-51, 1999.

[9] C. Augustin, V. Freib, E. Perrier, A. Huc, and O. Damour, "A skin equivalent model for cosmetological trials: an in vitro efficacy study of a new biopeptide," Skin Pharmacology, vol. 10, no. 2, pp. 63-70, 1997.

[10] T. S. Karande, J. L. Ong, and C. M. Agrawal, "Diffusion in musculoskeletal tissue engineering scaffolds: design issues related to porosity, permeability, architecture, and nutrient mixing," Annals of Biomedical Engineering, vol. 32, no. 12, pp. 1728-1743, 2004.

[11] S. W. Suh, J. Y. Shin, J. Kim et al., "Effect of different particles on cell proliferation in polymer scaffolds using a solvent-casting and particulate leaching technique," ASAIO Journal, vol. 48, no. 5, pp. 460-464, 2002.

[12] C. J. Coester, K. Langer, H. Von Briesen, and J. Kreuter, "Gelatin nanoparticles by two step desolvation: a new preparation method, surface modifications and cell uptake," Journal of Microencapsulation, vol. 17, no. 2, pp. 187-193, 2000.

[13] A. Saxena, K. Sachin, H. B. Bohidar, and A. K. Verma, "Effect of molecular weight heterogeneity on drug encapsulation efficiency of gelatin nano-particles," Colloids and Surfaces B: Biointerfaces, vol. 45, no. 1, pp. 42-48, 2005.

[14] F. Yanagawa, Y. Onuki, M. Morishita, and K. Takayama, "Involvement of fractal geometry on solute permeation through porous poly (2-hydroxyethyl methacrylate) membranes," Journal of Controlled Release, vol. 110, no. 2, pp. 395-399, 2006.

[15] J. Zhang and N. A. Peppas, "Synthesis and characterization of $\mathrm{pH}$ - and temperature-sensitive poly(methacrylic acid)/poly $(\mathrm{N}$ isopropylacrylamide) interpenetrating polymeric networks," Macromolecules, vol. 33, no. 1, pp. 102-107, 2000.

[16] R. C. Thomson, M. J. Yaszemski, J. M. Powers, and A. G. Mikos, "Hydroxyapatite fiber reinforced poly $(\alpha$-hydroxy ester) foams for bone regeneration," Biomaterials, vol. 19, no. 21, pp. 19351943, 1998.

[17] K. Whang, D. R. Elenz, E. K. Nam et al., "Engineering bone regeneration with bioabsorbable scaffolds with novel microarchitecture," Tissue Engineering, vol. 5, no. 1, pp. 35-51, 1999.

[18] T. Dutta Roy, J. L. Simon, J. L. Ricci, E. D. Rekow, V. P. Thompson, and J. R. Parsons, "Performance of degradable composite bone repair products made via three-dimensional fabrication techniques," Journal of Biomedical Materials Research A, vol. 66, no. 2, pp. 283-291, 2003.

[19] B.-S. Kim and D. J. Mooney, "Development of biocompatible synthetic extracellular matrices for tissue engineering," Trends in Biotechnology, vol. 16, no. 5, pp. 224-229, 1998.

[20] D. J. Mooney, C. L. Mazzoni, C. Breuer et al., "Stabilized polyglycolic acid fibre-based tubes for tissue engineering," Biomaterials, vol. 17, no. 2, pp. 115-124, 1996.

[21] J. P. Vacanti, "Beyond transplantation. Third annual Samuel Jason Mixter Lecture," Archives of Surgery, vol. 123, no. 5, pp. 545-549, 1988.
[22] J. P. Vacanti, M. A. Morse, W. M. Saltzman, A. J. Domb, A. Perez-Atayde, and R. Langer, "Selective cell transplantation using bioabsorbable artificial polymers as matrices," Journal of Pediatric Surgery, vol. 23, no. 1, pp. 3-9, 1988.

[23] A. S. Goldstein, G. Zhu, G. E. Morris, R. K. Meszlenyi, and A. G. Mikos, "Effect of osteoblastic culture conditions on the structure of poly(DL-lactic-co-glycolic acid) foam scaffolds," Tissue Engineering, vol. 5, no. 5, pp. 421-433, 1999.

[24] P. McPhie, "Dialysis," Methods in Enzymology, vol. 22, pp. 23-32, 1971. 

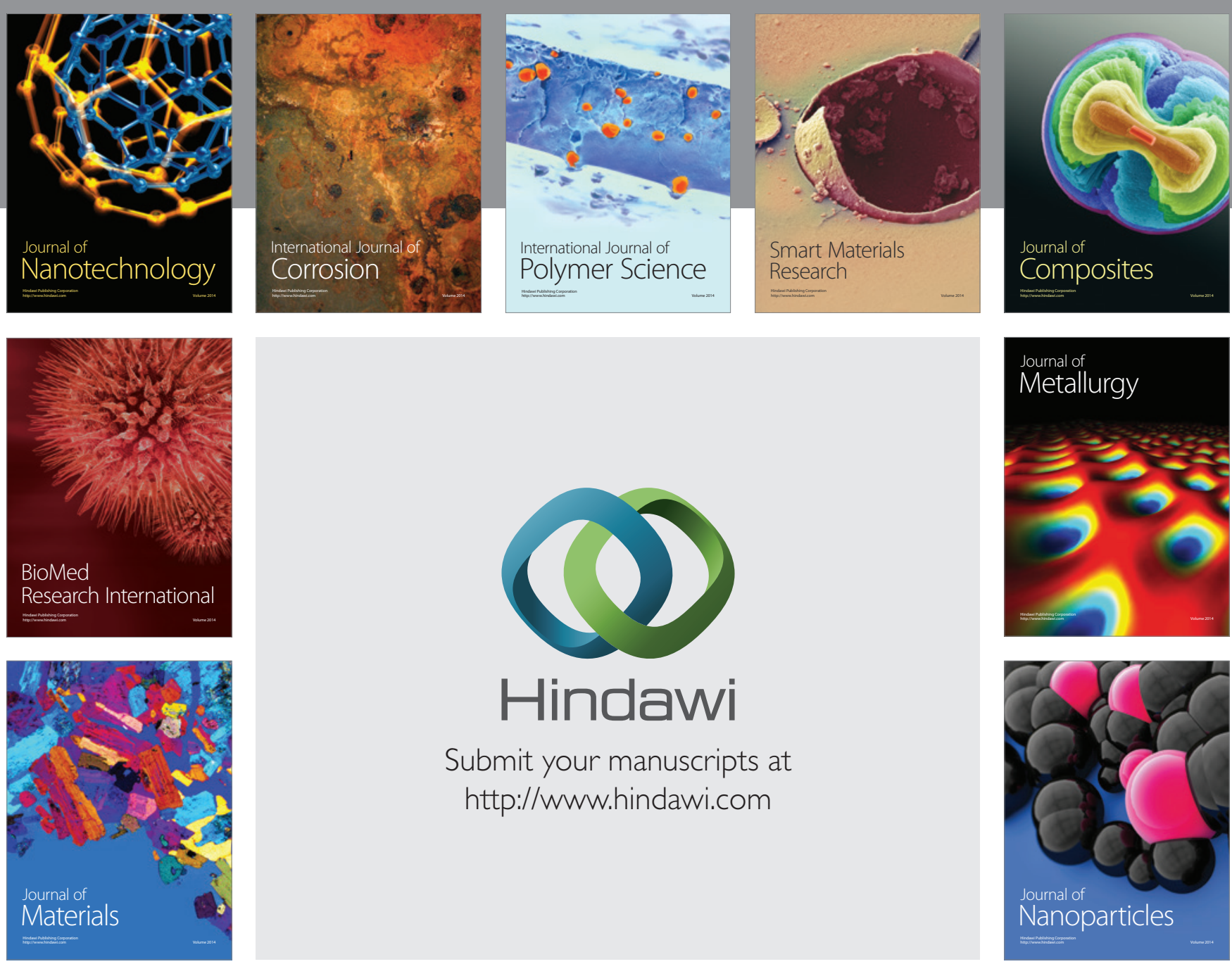

Submit your manuscripts at http://www.hindawi.com
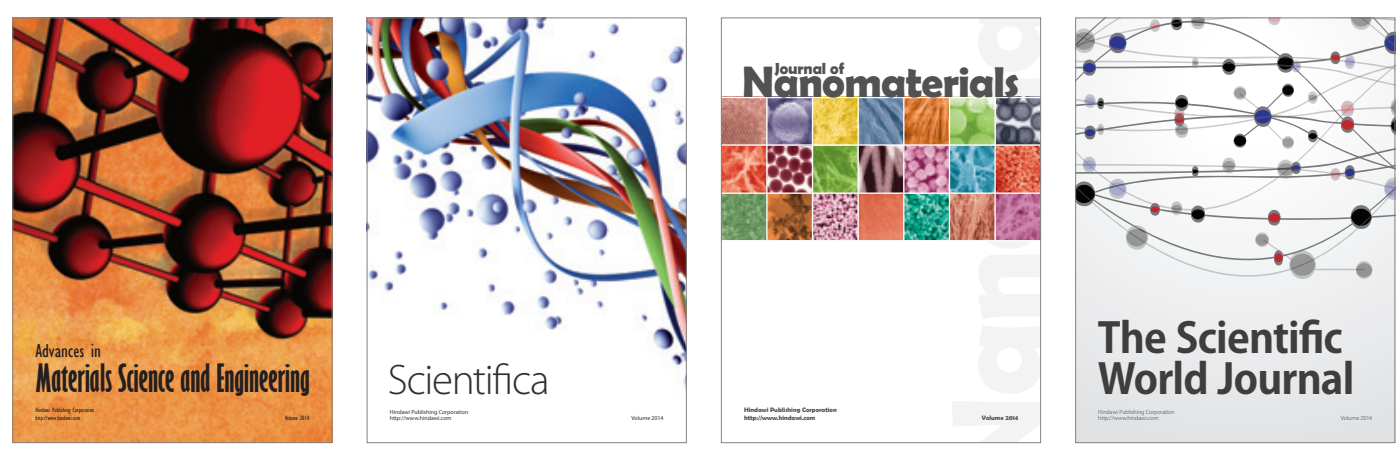

\section{The Scientific World Journal}
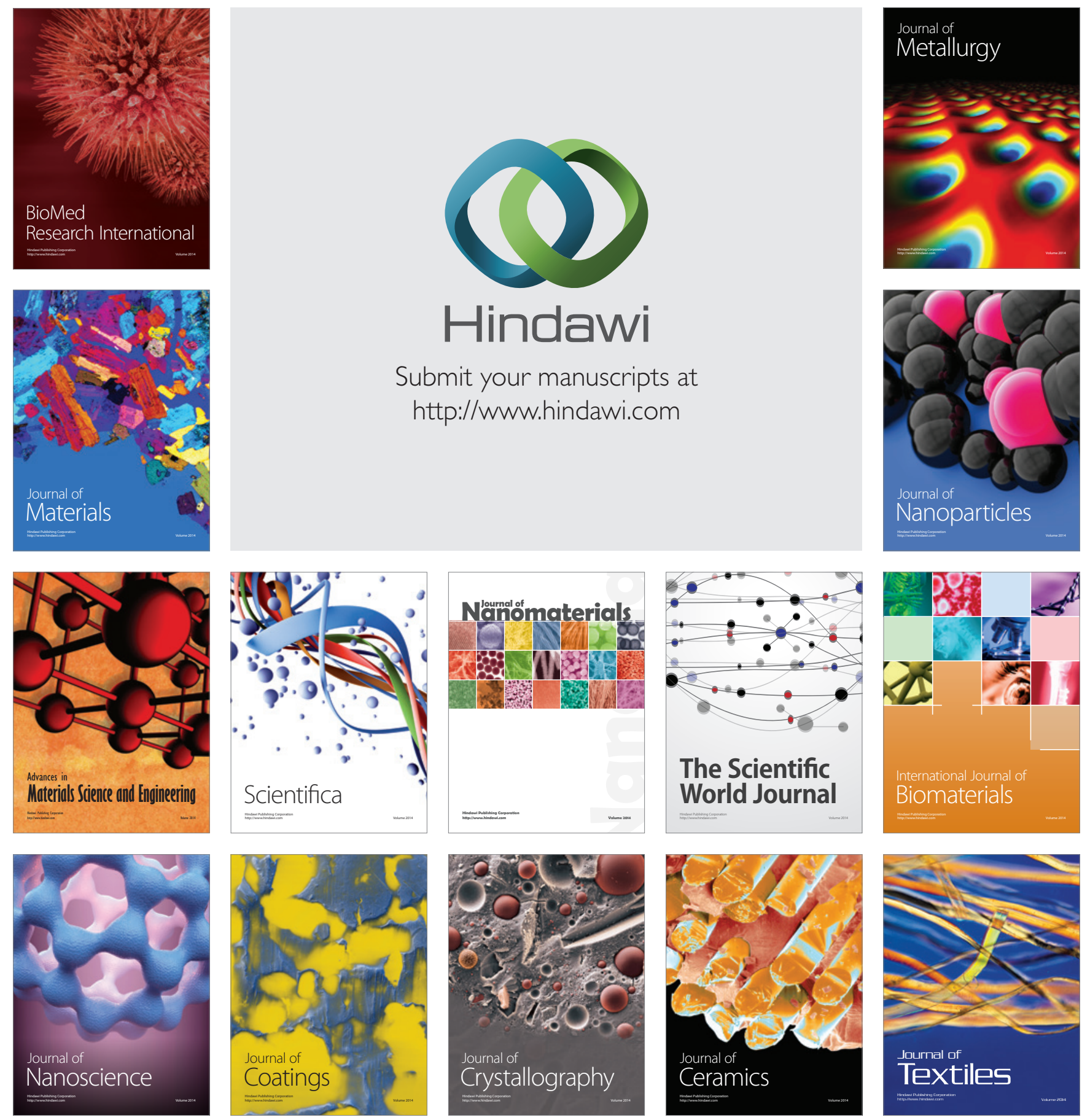